\title{
Formação e produção de conhecimento na universidade: estratégias de graduandos e pós-graduandos para a inserção no campo
}

\author{
Maísa Aparecida Oliveira* \\ Maria Cristina Silveira Galan Fernandes**
}

\section{Resumo}

O artigo busca compreender a formação e o processo de produção do conhecimento de estudantes da graduação e pós-graduação no contexto de intensificação da produtividade acadêmica por meio da análise de suas estratégias e ações. A pesquisa foi realizada com estudantes de uma universidade pública, tem caráter qualitativo, e a análise dos dados é fundamentada em Bourdieu e em perspectivas críticas de educação. A escolha dos estudantes por atividades legitimadas pela universidade fundamenta-se na instrumentalidade do sucesso escolar. Constatou-se que as atividades de pesquisa são essenciais para a formação acadêmica, profissional e pessoal do estudante, entretanto sua prática científica tem se mostrado intensificada e produtivista, orientando-se pela mercantilização do conhecimento. A formação dos universitários é pautada pela hegemonia da sociabilidade produtiva e pela normalização do produtivismo, indicando reconhecimento da hierarquia, subsumindo-se às regras como estratégia de inserção no campo acadêmico.

Palavras-chave: Formação acadêmica. Produção de conhecimento. Campo científico.

\footnotetext{
* Doutora pela Universidade Federal de São Carlos (UFSCar). Professora do departamento de Educação da Universidade do Estado de Minas Gerais (UEMG).

** Doutora pela Universidade Estadual Paulista (UNESP). Professora do departamento de Educação da Universidade Federal de São Carlos (UFSCar).

PERSPECTIVA, Florianópolis, v. 35, n. 2, p. 658-671, abr./jun. $2017 \quad$ http://www.perspectiva.ufsc.br
} 
Este artigo tem como objetivo refletir sobre a formação do estudante e o processo de produção do conhecimento no atual contexto de intensificação da produtividade acadêmica por meio da análise das estratégias e ações utilizadas por estudantes do ensino superior em nível de graduação e pósgraduação para inserção no campo acadêmico-científico.

Os dados apresentados neste artigo resultam de uma investigação iniciada no mestrado, já concluído, e continuada no doutoramento, em andamento, realizada com estudantes de uma universidade pública brasileira situada no estado de São Paulo. A coleta de dados foi realizada por meio da aplicação de questionários para estudantes de graduação e pós-graduação dos Centros de Ciências relacionados às áreas Biológicas, Humanas e Exatas e entrevistas semiestruturadas com estudantes da graduação.

Os dados quantitativos obtidos pelos questionários foram tratados estatisticamente, após o que se procedeu à análise de conteúdo para a compreensão dos significados (análise temática) e dos significantes (análise dos processos) representativos dos estudantes inseridos no campo universitário (BARDIN, 1977). A discussão dos dados obtidos na pesquisa é embasada na teoria de Pierre Bourdieu e em perspectivas críticas de educação.

Destaca-se nesta pesquisa a relevância do papel que os sujeitos (estudante da graduação e da pós-graduação) ocupam nos processos formativos e de produção do conhecimento na lógica social na qual estão inseridos, considerando as transformações ocorridas no interior da universidade.

\section{Interfaces da socialização do estudante universitário e do campo acadêmico- científico}

Neste trabalho, em que buscamos analisar o processo formativo e a produção de conhecimento de estudantes universitários (graduandos e pós-graduandos) caracterizados na perspectiva do produtivismo acadêmico, a teoria elaborada por Pierre Bourdieu, em especial a concepção de campo, apresenta-nos uma importante reflexão sobre as estratégias de socialização e inserção no campo acadêmico-científico vivenciadas pelos sujeitos. As relações que se estabelecem no campo, em uma disputa de posição que o estudante assume nos espaços sociais, constituem inter-relações entre a construção simbólica, a disposição social e a socialização do estudante no contexto universitário.

Para Bourdieu (1983b), o conceito de campo se refere às posições assumidas pelos sujeitos nos espaços sociais, indicando a compreensão de certos espaços de disposições sociais, nos quais 
determinado tipo de bem é produzido, consumido e classificado. Tal concepção representa o espaço social de dominação e de conflitos, cada qual detentor de sua autonomia e de suas próprias regras de organização e hierarquia social.

As relações de força que atuam no campo detêm seu objeto de disputa. Com base em Bourdieu (1983b), o objeto de disputa do campo científico é o monopólio da autoridade científica definida intrinsecamente como aptidão técnica e domínio social ou o monopólio da competência científica enquanto habilidade de falar e de agir legitimamente, o que é socialmente outorgado a um agente determinado.

Para Bourdieu (2004), todo campo se constitui como espaço de forças e de lutas para manter ou modificar tal campo de forças. O campo só existe pelos agentes e pelas relações objetivas estabelecidas entre os agentes pertencentes a determinado campo.

Bourdieu (2004) considera, ainda, as influências das relações que se estabelecem no campo e seu espaço de atuação. No campo científico é importante conhecer os pontos de vista de quem conduz as comunicações científicas, as temáticas e objetos pesquisados e os espaços de publicação, estabelecendo-se, assim, a estrutura das relações objetivas dos sujeitos que compõem o campo científico. $\mathrm{O}$ autor afirmou que tal estrutura das relações objetivas influencia no que os agentes podem ou não realizar, tomando como referência a posição ocupada pelo agente no campo, ou seja, a origem e a condição de classe a que pertence. A estrutura do campo científico é determinada pelo volume do capital de seu agente em proporção ao seu prestígio.

Segundo Bourdieu (2004), o indivíduo inserido no campo está em constante luta para se manter na posição adquirida em lutas precedentes, asseguradas pelo monopólio da autoridade científica. Tal autoridade está relacionada ao conhecimento da posição que este sujeito ocupa nas hierarquias instituídas (BOURDIEU, 2004), o que nos permite compreender a autoridade científica atribuída socialmente aos estudantes, bem como as condições e estratégias que estes agentes exercem no ensino superior.

Nota-se que o habitus adquirido pelo estudante condiciona diretamente suas ações no campo. Assim, para Bourdieu (1983a) a noção de habitus se refere ao sistema de disposições duráveis, estruturadas segundo o meio social dos sujeitos de disposições estruturadas (no social) e estruturantes (nas mentes), como princípio gerador e estruturador das práticas e das representações. O habitus é compreendido como referência das predisposições duráveis do modo de pensar, agir e portar-se. O 
habitus é incorporado nas relações estabelecidas socialmente, caracterizadas nas predileções e disposições dos indivíduos (NOGUEIRA, C.; NOGUEIRA, M., 2002).

A construção do habitus, enquanto estruturas estruturadas e estruturantes, como sistema de disposições socialmente constituídas, associa o princípio gerador e unificador do conjunto das práticas e das ideologias inerentes a um determinado grupo de sujeitos, o que condiciona uma posição e uma trajetória balizada no núcleo de um campo intelectual que, nesse sentido, ocupa um espaço deliberado na composição da classe dominante (BOURDIEU, 1982). Especificamente, a atuação do estudante na universidade conduz à reflexão e à atualização das disposições sociais e das distinções estruturais que são definidas, tornando-se assim parte distintiva de sua subjetividade.

A origem social e cultural dos sujeitos torna-se, portanto, elemento esclarecedor de suas trajetórias escolares, estratégias e das possibilidades de relações e posições hierárquicas que serão estabelecidas no campo. Tais relações ultrapassam a suposta neutralidade atribuída como um valor agregado e idealizado à instituição escolar.

Nesse sentido, podemos dizer que as diversas classes e fragmentos de classes desempenham uma luta especificamente simbólica para outorgar a definição do mundo social. Atendem, assim, aos seus interesses e impõem, no campo das tomadas de posições ideológicas, a reprodução da configuração transfigurada ao campo das posições sociais. Nesse enredo, Bourdieu (1983a, p. 12) afirma que "O campo de produção simbólica é um microcosmos da luta simbólica entre as classes".

Para Bourdieu (1983a), a posição que o indivíduo ocupa nos diferentes campos define a sua posição no espaço social, ou seja, na disposição dos poderes que exerce em cada campo, discriminado pelos capitais social, econômico e simbólico, o espaço social do indivíduo é deliberado. A posição no campo social determina os poderes atuais ou potenciais nos diferentes campos, além de oportunizar as possibilidades futuras de acesso aos capitais simbólico, social e cultural. De maneira particular, o capital econômico tende a impor a sua estrutura a cada campo e à hierarquia que se estabelece nele. Com base em tal discussão, compreendemos que o estudante universitário detentor dos capitais legitimados pela universidade ocupa uma posição social privilegiada em relação aos estudantes pertencentes às classes sociais dominadas, que não detêm, ou detêm apenas parcialmente, os capitais legitimados pela universidade. A posição que o estudante ocupa antes de ingressar na universidade tende a ser reproduzida no campo acadêmico.

Nesse sentido, as escolhas dos estudantes no campo acadêmico não são isentas de parcialidade, pois as escolhas por uma prática social são produto das condições de socialização em uma determinada estrutura social. 
Consideramos, portanto, que Bourdieu nos revela elementos e categorias fundamentais para a análise de dados referentes à condição social, econômica e escolar do estudante universitário, nosso sujeito de estudo, a qual se reproduz objetivamente nos processos de socialização, de formação e de atuação destes estudantes.

\section{A universidade e sua política mercadológica: cenário formativo do estudante de graduação e pós-graduação}

O período atual de regulação do capitalismo, em um contexto de exigências diferenciadas, designa demandas e desafios à educação, de maneira peculiar à educação superior, incluindo os processos de internacionalização e globalização da economia. O modo de produção capitalista tem como princípio primeiro de sustentação a necessidade de permanentes transformações visando atender a um mercado cada vez mais competitivo. Assim, há uma maneira específica de compreensão da ampliação do capital estabelecida nas relações com o trabalho, nas quais a ciência e a tecnologia são concebidas como força de produção, em uma lógica racional que tem como paradigma os princípios da economia privada.

Como afirmam Oliveira e Catani (2011), o sistema econômico e as ações governamentais (com interesses políticos) exercem papel de dominação sobre o campo da educação superior, que de maneira peculiar exerce, sobretudo, dois papéis significativos no processo de acumulação da mundialização do capital, a saber: formar profissionais aptos a atuar e a desenvolver atividades altamente qualificadas no mercado de trabalho e gerar conhecimentos novos e inovações tecnológicas capazes de exercer competitividade nos diversos setores da economia.

Em função das mudanças recentes no campo da educação superior, problemas e embates surgem no campo acadêmico-científico. A universidade, cada vez mais atrelada à mundialização do capital, precisa desenvolver pesquisas que agreguem maior valor econômico, a fim de que o investimento feito pelo Estado e/ou pelo mercado tenha retorno satisfatório, respondendo às regulamentações, às demandas e aos controles de qualidade externos aos agentes institucionais e ao próprio campo científico.

Dominantes e dominados no campo universitário poderão, cada vez mais, se submeter à lógica hegemônica da mundialização do capital, à chamada economia do conhecimento, à lógica e ao processo de reconfiguração da educação superior. $\mathrm{O}$ campo universitário vem, pois, sendo levado pelas políticas públicas de educação 
superior a subordinar-se a uma determinada lógica externa, perdendo paulatinamente a autonomia para se autodefinir, o que se dá em grande parte pela intensificação de sua própria natureza, como espaço de luta pela distinção universitária, autoridade científica e poder acadêmico-científico. (OLIVEIRA; CATANI, 2011, p. 19).

Tais questões sobre o atual contexto da política de educação superior no Brasil nos permitem inferir sobre a mudança na identidade e cultura da universidade, especificamente nas relações que a instituição universitária estabelece com a sociedade e, principalmente, com o Estado. Essas relações atuam diretamente no trabalho do professor, como já assinalado por Sguissardi e Silva Júnior (2009); Silva Júnior, Sguissardi e Silva (2010); Silva e Silva Júnior (2010). Assim, torna-se fundamental a problematização da formação do estudante universitário, tanto na graduação quanto na pós-graduação, quando as pesquisas desenvolvidas por este estudante junto aos professores geram inovações tecnológicas e científicas que favorecem a industrialização do conhecimento.

Nesse sentido, entende-se que a formação de estudantes universitários para a cidadania e para o mundo do trabalho necessita de critérios de qualidade compatíveis com as exigências estabelecidas pelo campo acadêmico.

Para Silva Júnior et al. (2013), a produção do conhecimento e a intensificação das atividades acadêmico-científicas impõem a cultura do produtivismo acadêmico, em que a pós-graduação se torna o polo irradiador da nova cultura institucional para toda a universidade pública.

A cultura da pós-graduação está enraizada na universidade, tendo em vista que mesmo os professores que não atuam neste nível de ensino anseiam ingressar na pós-graduação para obterem melhores condições e oportunidades de financiamento para pesquisa. Da mesma maneira, os professores que atuam na pós-graduação objetivam se manter no campo, o que perpetua a cultura produtivista da pós-graduação, realizando pesquisas de iniciação científica (IC) e ministrando aulas que resultem em publicações (SILVA JÙNIOR et al., 2013). Assim, a pós-graduação amplia, na universidade, uma submissão ao aparelho econômico global e concorrente de mercados.

Diante do exposto, é preciso repensar as saídas para a universidade enquanto instituição social e, de modo específico, os dilemas do estudante da graduação e da pós-graduação na universidade pública brasileira. Assim, problematizamos em nossa pesquisa o processo de constituição da atuação deste estudante em uma sociabilidade produtiva/produtivista no campo acadêmico. E buscamos analisar o impacto do produtivismo acadêmico experienciado pelo estudante nos processos de formação e de produção de conhecimento regulados no modelo de avaliação mercantil proposto para o Ensino Superior. 


\section{Atuação e estratégias de inserção do estudante universitário no campo}

Utilizamos neste trabalho os resultados de questionários respondidos pelos estudantes da pósgraduação e da graduação e entrevistas que foram realizadas, até o momento, com os estudantes da graduação. A construção dos dados abarcou questões sobre o perfil socioeconômico, a trajetória escolar, processo formativo, inserção e participação em grupos sociais e universitários, relações estabelecidas na universidade, estratégias de atuação no campo acadêmico e autoavaliação da atuação do discente.

Em uma breve caracterização dos participantes da pesquisa, verificamos dentre a amostra dos estudantes da graduação (120 estudantes) que a média das idades foi compreendida em 23 anos, 57\% dos estudantes se declaram do sexo feminino, $54 \%$ dos participantes frequentaram escolas privadas em sua formação básica e parcela significativa dos pais dos estudantes são detentores de altos níveis de escolarização (ensino superior e pós-graduação).

Já em nível de pós-graduação, a amostra foi constituída de 45 estudantes do mestrado e 60 estudantes do doutorado, totalizando 105 pós-graduandos. Os dados dos questionários indicaram que a média de idades foi de 33 anos, e $80 \%$ dos estudantes são do sexo feminino. 52\% dos participantes frequentaram grande parte do Ensino Básico em escolas públicas, e 69\% fizeram a graduação em instituições públicas de Ensino Superior. Observamos que os pais são escolarizados e que parte expressiva deles possui graduação e pós-graduação.

Embasando-se no sociólogo Lahire (1998), Setton (2005) faz menção ao processo de socialização do sujeito, que pode ser identificado de maneira plural. Podemos compreender a pluralidade nas diferentes relações estabelecidas pelo estudante na universidade, que são concebidas devido às diversas posições sociais por ele ocupadas, exprimindo experiências variadas e, até mesmo, conflitantes, caracterizadas pela heterogeneidade de princípios de ação.

Compreendemos, assim, as relações estabelecidas na universidade como aquelas que constroem o campo acadêmico. Os dados da pesquisa revelaram que a relação estabelecida entre os estudantes da graduação foi intermediada pelo objetivo acadêmico e caracterizada de modo impessoal pelo distanciamento entre os próprios estudantes. Os graduandos se referiram à competição existente no campo acadêmico, que, apesar de estar presente na sociedade, pode ser identificada, especificamente, entre os estudantes que almejam ingressar na pós-graduação. 
Os estudantes da pós-graduação consideraram o espaço acadêmico por muitas vezes privilegiado para estabelecer vínculos de amizades e já receberam (ou ofereceram) auxílio em alguma atividade acadêmica, apesar de também apontarem a existência de conflitos entre pós-graduandos. Uma parcela significativa dos participantes da pesquisa afirmou ainda que a competitividade é uma constante entre os pós-graduandos.

A relação estabelecida entre os estudantes e professores é fundamental no processo formativo dos estudantes. Constatamos que os professores são referências para os estudantes, graduandos e pósgraduandos, no que se refere à formação acadêmica, ao processo de produção de conhecimento e à maneira de se fazer pesquisa.

A universidade é um espaço privilegiado para se conhecer várias pessoas com culturas diferentes, em que ocorre constantemente trocas de conhecimentos e experiências entre estudantes, professores, técnicos e outros.

O aprendizado e as trocas de experiências para os estudantes universitários ocorrem em atividades que não são consideradas acadêmicas. Entendemos por atividades não acadêmicas as relacionadas aos movimentos estudantis (Diretório Central de Estudantes - DCE, Centro Acadêmico CA e outros) e aos grupos informais (esportivos, artísticos e outros). Analisadas todas as respostas dos estudantes, podemos constatar que os graduandos e pós-graduandos não participaram de muitas atividades desvinculadas da Academia.

Em relação às atividades acadêmicas, os estudantes da graduação referiram-se à participação em disciplinas, estágios curriculares e extracurriculares, iniciação científica, projetos de extensão, grupos de pesquisas, entre outros. Uma estudante de graduação entrevistada decidiu já no primeiro ano do curso de Biotecnologia que iria prosseguir a carreira acadêmica, teve como objetivo, a partir de então, preencher o Currículo Lattes.

O capital cultural incorporado pelos estudantes no decorrer de sua trajetória universitária incide em suas escolhas, o que sugere a propensão de investir nas atividades legitimadas pela universidade (campo dominante), isto é, as atividades mais "rentáveis". Com base em Bourdieu (1983a), apreendemos que as atividades rentáveis estão diretamente relacionadas ao campo científico que determina (rege) a Ciência. As atividades rentáveis possibilitam ao estudante a obtenção do reconhecimento de seus pares. Em face à preferência dos graduandos e pós-graduandos pelas atividades acadêmicas, constatamos que as atividades são compreendidas como rentáveis para o campo universitário. Ao realizarem as atividades legitimadas pela instituição universitária, optam pela 
instrumentalidade do sucesso escolar, categoria constitutiva para o ingresso e a legitimidade do estudante no campo acadêmico-científico.

Segundo os estudantes de graduação entrevistados, a atividade mais significativa que realizaram no período da graduação está relacionada à pesquisa. Tal cenário institucional incide na formação do universitário, quando se observa a existência de uma continuidade progressiva no campo universitário no decurso da graduação para a pós-graduação, em que o interesse pela pesquisa faz com que o estudante queira prosseguir a carreira acadêmico-científica (OLIVEIRA; FONSECA, 2010).

$\mathrm{O}$ estudante que participa de atividades relacionadas à pesquisa acadêmica desenvolve a capacidade de produzir seu próprio conhecimento, além do aprendizado que adquire para relacionar a teoria e a prática (FERNANDES, 2002). Também Fava-de-Moraes e Fava (2000) consideram que a IC proporciona ao estudante o desenvolvimento de sua análise crítica, compreensão da lógica científica, além da possibilidade de inserção em programas de pós-graduação.

Verificamos que a prática de iniciação científica remunerada pode contribuir para a manutenção do estudante na universidade e também estimular investimentos em sua própria formação: na aquisição de livros, materiais e na realização de cursos de línguas estrangeiras.

No entanto, segundo Santos (2013), a prática de pesquisa na graduação está se transformando em um novo paradigma. Para a autora, a pesquisa desenvolvida pelo estudante na graduação é importante para o processo formativo, por outro lado aprofunda princípios como o de competitividade e de individualismo no sujeito. Considera que a prática acadêmica de pesquisa que o estudante da graduação vivencia na universidade está associada ao produtivismo acadêmico, como materialização da racionalidade econômica imersa no campo acadêmico-científico. Santos (2013) compreende que os programas de iniciação à pesquisa possuem a função de preparar o estudante para o mestrado e o doutorado, ao constatar que a pesquisa científica realizada na graduação mantém os métodos de produção e reprodução do conhecimento presentes na pós-graduação, o que também foi identificado em nossas pesquisas.

O financiamento das pesquisas realizadas na universidade, segundo Sguissardi e Silva Júnior (2009), possuem o objetivo de favorecer a crescente mercantilização da produção do conhecimento, o que implica na controversa representação do estudante da graduação como mão de obra barata e qualificada. O estudante, ao ser considerado como mão de obra, é avaliado e cobrado como tal em suas atividades de Pesquisa, Ensino e Extensão. Tais atividades, de acordo com a função social da 
universidade, deveriam se caracterizar unicamente como formativas, sem as exigências da mercantilização do conhecimento.

Os estudantes da graduação relacionaram suas produções aos benefícios sociais ou à contribuição dada à sua área de pesquisa, o que também é função social da universidade, ou seja, contribuir com a resolução dos problemas e produzir Ciência. No entanto, os universitários não se referiram às contribuições que suas atividades proporcionaram às empresas e/ou ao Estado (SGUISSARDI; SILVA JÚNIOR, 2009). Nesse sentido, nos questionamos se os estudantes estão desenvolvendo as pesquisas sem refletir adequadamente sobre todas as dimensões de sua aplicabilidade.

É possível perceber a crescente produção científica do estudante da graduação e da pósgraduação. O estudante da graduação está aprendendo a produzir trabalhos científicos e, como já exposto, o professor-orientador é a referência no aprendizado em tal processo. Embasando-nos em Sguissardi e Silva Júnior (2009), inferimos que o professor universitário imerso na lógica produtivista tem seu trabalho intensificado, pautando-se no produtivismo acadêmico, em que a quantidade da produção científico-acadêmica é demasiadamente valorizada. Uma estudante demonstrou em seus relatos que, na graduação, assim como seu orientador, incorporou uma prática de produção acadêmica que segue a lógica do trabalho intensificado dos docentes universitários, adquirindo uma concepção ansiosa de como fazer pesquisa e a prática intensificada de produção do conhecimento.

A intensificação do trabalho universitário também é identificada no estudante da pósGraduação. Os participantes da pesquisa dedicam às atividades da pós-graduação, em média, entre seis e dez horas diárias, além de uma parcela expressiva dos estudantes relatarem que dedica mais de dez horas diárias à pós-graduação.

A intensificação do trabalho do pós-graduando pode ser observada na recorrente colaboração que existe entre os estudantes e os professores-orientadores, identificada nas produções intelectuais (publicações), atividades de pesquisa, de ensino e de orientação a estudantes de outros níveis de ensino.

Outro fator que nos permite inferir que as atividades desenvolvidas pelos estudantes da pósgraduação seguem a lógica do trabalho intensificado se constata na cobrança por publicações, defesa de teses e dissertações no período previsto, participação em eventos com apresentação de trabalhos e obtenção de dados de pesquisa com elevado impacto social e acadêmico.

Para Silva Júnior et al. (2013), os estudantes que ingressam na pós-graduação após a vigência do atual sistema de avaliação da Coordenação de Aperfeiçoamento de Pessoal de Nível Superior (Capes), implantado a partir de 1997 e intensificado com o passar dos anos, mostram-se mais adaptados PERSPECTIVA, Florianópolis, v. 35, n. 2, p. 658-671, abr./jun. $2017 \quad$ http://www.perspectiva.ufsc.br 
ao produtivismo acadêmico, ao individualismo e à competitividade. Segundo as pesquisas realizadas por Sguissardi e Silva Júnior (2009), o estudante da pós-graduação também é um agoniado do sistema de avaliação da Capes. Isto porque a lógica produtivista é naturalizada pelo professor-pesquisador ao compactuar com as relações intensificadas entre a universidade e o mercado, modificando os rumos da formação dos pós-graduandos.

A universidade, imersa nessa lógica intensificada, espera um habitus específico para o seu aluno e, muitas vezes, exclui os estudantes que não tiveram acesso aos capitais social, familiar, econômico e escolar para a obtenção do habitus acadêmico exigido. Assim, os estudantes precisam incorporar o habitus esperado pela universidade e se integrar à lógica produtivista, como constatado pelos participantes da pesquisa.

A hierarquização presente no campo científico nos faz problematizar o processo de publicação dos trabalhos produzidos pelos estudantes, graduandos e pós-graduandos, pois define o acesso a determinados meios de publicações e tal hierarquia se efetiva devido à posição ocupada no campo. Os estudantes da graduação revelaram que há espaços científicos em que são impossibilitados de divulgar suas pesquisas e indicaram também que, quando o trabalho científico é realizado em conjunto com estudantes da pós-graduação ou com professores, existe a possibilidade de aprovação do trabalho, ratificando a hierarquização no campo acadêmico e reconhecendo a posição social que ocupam.

A partir da compreensão da teoria elaborada por Bourdieu (1983b), consideramos que no campo científico existe uma hierarquia social presente em todas as instâncias. Tal hierarquização define as práticas e escolhas, constituindo, assim, a hierarquia social, nesse caso, de quem pode ter acesso a determinados meios de publicação e de suas estratégias.

Para Bourdieu (1983b), os dominantes no campo científico utilizam estratégias para se manterem nas posições ocupadas no campo, usufruindo de estratégias de conservação, a fim de ratificar a ordem científica instituída, com a qual compactuam. Os novatos se utilizam de estratégias de sucessão que acarretam princípios de legitimação da dominação. As estratégias dos estudantes podem ser identificadas na pesquisa como promissoras e importantes. Se o estudante (novato) ainda não conseguiu se inserir no campo científico, pode usar estratégias ao desenvolver uma boa pesquisa em colaboração com o orientador (já consolidado no campo), que permitirá o acesso a uma posição privilegiada no campo científico. 


\section{Considerações finais}

Neste trabalho, buscamos trazer questionamentos e reflexões sobre a formação e a produção de conhecimento de estudantes da graduação e da pós-graduação.

Com base nos resultados da pesquisa, constatamos que a formação do estudante tem se caracterizado na escolha pelas atividades legitimadas pela universidade (campo dominante), que lhes são rentáveis academicamente e que possibilitam a obtenção do reconhecimento dos seus pares.

A imersão do estudante universitário na lógica acadêmica produtivista acarretou a intensificação das atividades, sobrecarregando o estudante com tarefas que são avaliadas pelas agências de fomento de suas pesquisas.

Constatamos que o estudante universitário assumiu a normalização do produtivismo, espelhando-se em seus professores, adaptando-se às regras, normas, práticas e processos do sistema de mercantilização do conhecimento, o que demonstra reconhecimento da hierarquia social, subsumindose a tais regras como estratégia de inserção no campo acadêmico-científico.

\section{REFERÊNCIAS}

BARDIN, L. Análise de Conteúdo. Lisboa: 70, 1977.

BOURDIEU, P. A economia das trocas simbólicas. São Paulo: Perspectiva, 1982.

BOURDIEU, P. Questões de sociologia. Rio de Janeiro: Marco Zero, 1983a.

BOURDIEU, P. O Campo Científico. In: ORTIZ, R. (Org.). Pierre Bourdieu - Sociologia. São Paulo: Ática, 1983b. p. 122-155.

BOURDIEU, P. Os usos sociais da ciência: por uma sociologia clínica do campo científico. São Paulo: UNESP, 2004.

FAVA-DE-MORAES, F.; FAVA, M. A iniciação científica: muitas vantagens e poucos riscos. São Paulo em Perspectiva, 14, 73-77, 2000. Disponível em: <http:// $<$ www.scielo.br/scielo.php?script=sci_arttext\&pid=S0102-88392000000100008\&lng=en\&nrm=iso $>$. Acesso em: 16 maio 2013. 
FERNANDES, M. C. S. G. Concepções de qualidade de ensino na perspectiva docente em um centro universitário privado e noturno. 2002. 339f. Tese (Doutorado em Educação) - Universidade Estadual Paulista, Araraquara, 2002.

NOGUEIRA, C. M. M.; NOGUEIRA, M. A. A sociologia da educação de Pierre Bourdieu: limites e contribuições. Educação e Sociedade, Campinas, v. 23, n. 78, p. 15-35, 2002.

OLIVEIRA, J. F.; CATANI, A. M. A reconfiguração do campo universitário no Brasil: conceitos, atores, estratégias e ações. In: OLIVEIRA, J. F. (Org.). O campo Universitário no Brasil: políticas, ações e processos de reconfiguração. Campinas: Mercado de Letras, 2011. p. 11-37.

OLIVEIRA, J. F.; FONSECA, M. A pós-graduação brasileira e seu sistema de avaliação. In: OLIVEIRA, J. F.; CATANI, A. M.; FERREIRA, N. S. C. (Orgs.) Pós-graduação e avaliação: impactos e perspectivas no Brasil e no cenário internacional. Campinas: Mercado de Letras, 2010. p. $15-52$.

SANTOS, S. A. Mudanças na graduação na universidade pública: a nova prática da Iniciação Científica. 2013. 126f. Tese (Doutorado em Educação) - Programa de Pós-Graduação em Educação, Universidade Federal de São Carlos, São Carlos, 2013.

SETTON, M. D. G. J. A particularidade do processo de socialização contemporâneo. Tempo Social, n. 17, p. 335-350, 2005. Disponível em <http://www.revistas.usp.br/ts/article/view/12482/14259>. Acesso em: 27 nov. 2013.

SGUISSARDI, V.; SILVA JÚNIOR, J. dos R. Trabalho intensificado nas federais: Pós-graduação e produtivismo acadêmico. São Paulo: Xamã, 2009.

SILVA, E. P E.; SILVA JÚNIOR, J. dos R. Estranhamento e desumanização nas relações de trabalho na instituição universitária pública. Revista HISTEDBR On-line, Campinas, Número Especial, 2010. p. 223-238.

SILVA JUNIOR, J. R. et al. Trabalho do professor pesquisador diante da expansão da pós-graduação no Brasil pós-LDB. Revista Brasileira de Educação. Rio de Janeiro, v. 18, n. 53, 2013. Disponível em: <http://www.scielo.br/scielo.php?script=sci_arttext\&pid=S141324782013000200011\&lng=en\&nr $\mathrm{m}=$ iso $>$. Acesso em: 6 ago. 2013.

SILVA JÚNIOR, J. R; SGUISSARDI, V.; SILVA, E. P. Trabalho intensificado nas federais públicas brasileiras. Universidade e Sociedade, Brasília, DF, n. 45, p. 9-37, 2010. 


\section{Education and Knowledge Production in the University: Strategies of undergraduate and graduate students for entering the field}

\begin{abstract}
The article tries to understand education and the knowledge production process of undergraduate and graduate students in the context of the intensification of academic productivity through the analysis of the students' strategies and actions. A survey was conducted with students from a public university. The research had a qualitative approach and data analysis was based on Bourdieu and on critical perspectives on education. The students' decision to choose activities legitimized by the university is based on the instrumentality of school success. It was found that research activities are essential for the academic, professional and personal education of students, however, their scientific practice has shown to be intensified and productivist, guided by the mercantilization of knowledge. The education of university students is based on the hegemony of productive sociability and the normalization of productivism, indicating recognition of the hierarchy, subsumed under rules as a strategy for insertion in the academic field.
\end{abstract}

Keywords: Academic education. Production of knowledge. Scientific field.

Maísa Aparecida Oliveira

E-mail:maisavrb@hotmail.com

Maria Cristina Silveira Galan Fernandes

E-mail: mcsgfernandes@gmail.com

\section{Formation et production de la connaissance} dans l'université: les stratégies des étudiants de licence, master e doctorat pour l'insertion dans le champ

\section{Resumé}

Cet article à l'objectif de comprendre la formation et le processus de production de la connaissance des étudiants de licence, master e doctorat dans le contexte d'intensification de la productivité universitaire à travers l'analyse de leurs stratégies et actions. La recherche développée avec des étudiants d'une université publique possède caractère qualitatif et l'analyse des données est fondée sur Bourdieu et sur les perspectives critiques d'éducation. La sélection des étudiants par des activités légitimées par l'université est fondée sur l'instrumentalité de la réussite scolaire. Nous avons vérifié que les activités de recherche sont essentielles pour la formation universitaire, professionnelle et personnelle de l'étudiant, pourtant sa pratique scientifique se révèle intensifiée et productiviste, guidée par la marchandisation de la connaissance. La formation des étudiants universitaires est guidée par l'hégémonie de la sociabilité productive et par la normalisation du productivisme. Cela indique la reconnaissance de la hiérarchie et la soumission aux règles comme stratégie d'insertion dans le domaine universitaire.

Mots-clés: Productivité Académique. Production de connaissances. Domaine scientifique.

Enviado em: 08/06/2015

Aprovado em: 28/02/2016 